\title{
Ten-year outcomes of an early invasive or a selective invasive strategy in non-ST-segment elevation acute coronary syndrome patients with and without diabetes mellitus: a subgroup analysis of the ICTUS trial
}

\author{
Niels P.G. Hoedemaker ${ }^{\mathrm{a}}$, Peter Damman ${ }^{\mathrm{a}, \mathrm{b}}$, Pier Woudstra ${ }^{\mathrm{a}}$, \\ Alexander Hirsch ${ }^{\mathrm{c}, \mathrm{d}}$, Fons Windhausen ${ }^{\mathrm{e}}$, Jan G. Tijssen ${ }^{\mathrm{a}}$ \\ and Robbert J. de Winter ${ }^{\mathrm{a}}$; on behalf of the ICTUS investigators
}

\author{
Coronary Artery Disease 2020, 31:95-97

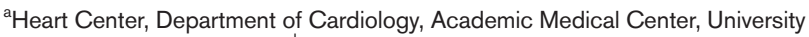 \\ of Amsterdam, Amsterdam, ' Department of Cardiology, Radboud University \\ Medical Center, Nijmegen, Departments of ${ }^{\mathrm{C}}$ Cardiology, ${ }^{\mathrm{d}}$ Radiology and Nuclear \\ Medicine, Erasmus MC, University Medical Center Rotterdam, Rotterdam and \\ e Department of Cardiology, Rode Kruis Ziekenhuis, Beverwijk, The Netherlands
}

Approximately $20 \%-25 \%$ of non-ST-segment elevation acute coronary syndrome (NSTE-ACS) patients have diabetes mellitus (DM) [1]. Patients with DM have worse outcomes due to higher rates of cardiovascular risk factors and coronary artery disease compared to non-diabetic patients [2]. The European and American guidelines recommend an invasive strategy with coronary angiography within 72 hours after presentation in patients with DM, whereas a selective invasive strategy remains reserved for lower risk NSTE-ACS patients [3,4]. These recommendations are based on meta-analysis with less than 9000 patients demonstrating a short- and medium-term reduction in MI and rehospitalisation at 1 year [5]. Longterm outcomes of an early or selective invasive strategy in NSTE-ACS patients with DM remain underreported. Therefore we assessed 10-year outcomes of an early or selective invasive strategy in NSTE-ACS patients with or without DM.

Between July 2001 and August 2003, 1200 NSTE-ACS patients enrolled in the Invasive Versus Conservative Treatment in Unstable Coronary Syndromes (ICTUS) trial were randomly assigned to an early invasive $(\mathrm{n}=$ 604 , angiography $<24-48$ hours after randomisation) or a selective invasive strategy [ $\mathrm{n}=596$, optimal (antianginal) medical therapy, angiography in case of refractory angina or inducible signs of ischemia during a pre-discharge ischemia detection test]. The study design of the ICTUS trial has been previously reported [6]. We collected and adjudicated 10 -year follow-up of death, myocardial infarction (MI), and revascularisation through the Dutch population registry, phone calls, and patient records. The main outcome was 10-year death or spontaneous MI.

At baseline 166 patients (14\%) were known with DM, of which 86 were randomised to an early invasive and 80 to a selective invasive strategy. Baseline characteristics were
Correspondence to Robbert J. de Winter, MD, PhD, FESC, Department of Cardiology, B2-137, Academic Medical Center Amsterdam, Meibergdreef 9 1105, Amsterdam, The Netherlands

Tel: +0031205669111; fax: +0031206962609; e-mail: r.j.dewinter@amc.uva.nl

Received 15 January 2019 Accepted 7 September 2019

evenly distributed between patients in the early or selective invasive groups, regardless of the presence of DM. However, diabetic patients in the early invasive group were older compared to the selective invasive group [69.0 years; interquartile range (IQR): $60.8-74.0$ vs. 65.5 years; IQR: 55.3-72.0; $P=0.025]$. Diabetic patients had higher rates prior MI ( $31 \%$ vs. $22 \%$, hypertension $(57 \%$ vs. $36 \%)$, ST-segment deviation (55\% vs. $47 \%$ ), and three-vessel and/or left main coronary artery disease ( $31 \%$ vs. $14 \%)$.

In-hospital angiography ( $75 \%$ vs. $77 \%, P=0.77)$ and revascularisation $(56 \%$ vs. $58 \%,=0.60)$ were similar in patients with and without DM. Among diabetic patients, in-hospital angiography was performed in $97.7 \%$ in the early invasive group and $53.8 \%$ in the selective invasive group $(P>0.001)$. In-hospital revascularisation among patients with DM was $70.9 \%$ and $40.0 \%$ in the early and selective invasive groups respectively $(P<0.001)$. Most diabetics patients were treated with percutaneous coronary intervention (early: $52.3 \%$ vs. selective: $30.0 \% ; P=0.004$ ). In-hospital coronary artery bypass grafting performance in diabetic patients was not significantly different between the two groups (early: $18.6 \%$ vs. selective: $10.0 \% ; 0.115$ ).

Patients with DM had worse 10-year clinical outcomes compared to patients without DM, as is displayed in Fig. 1. Death or spontaneous MI at 10-year was significantly higher in diabetic patients $(51.7 \%)$ vs. nondiabetic patients (28.1\%) [hazard ratio (HR): 2.26; 95\% confidence interval (CI): 1.77-2.88; $P<0.001$ ]. All other events also were significantly higher among diabetic patients, with the exception of first revascularisation and procedure-related MI. Among patients with DM, 10-year death or spontaneous MI was 58.5\% in the early invasive group and $44.4 \%$ in the selective invasive group (HR: $1.44 ; 95 \%$ CI: $0.93-2.22 ; P=$ 0.101 ) (Table 1). There were no significant differences 


\begin{tabular}{|c|c|c|c|c|c|c|c|c|}
\hline & $\begin{array}{l}\text { Patients with DM } \\
(n=166)\end{array}$ & $\begin{array}{l}\text { Patient without DM } \\
(n=1034)\end{array}$ & HR & Cl $95 \%$ & & & & p-value \\
\hline Death or spontaneous MI & $84(51.7)$ & $283(28.1)$ & 2.26 & $(1.77-2.88)$ & & & $\rightarrow-$ & $<0.001$ \\
\hline Death or MI & $86(52.9)$ & $313(31.0)$ & 2.02 & $(1.59-2.57)$ & & & - & $<0.001$ \\
\hline All-cause death & $72(44.7)$ & $222(22.0)$ & 2.41 & $(1.85-3.15)$ & & & - & $<0.001$ \\
\hline Cardiovascular death & $48(32.4)$ & $134(13.9)$ & 2.66 & $(1.91-3.70)$ & & & $\longrightarrow$ & $<0.001$ \\
\hline Non-cardiovascular death & $24(18.2)$ & $88(9.4)$ & 2.04 & $(1.30-3.20)$ & & & $\longrightarrow$ & 0.002 \\
\hline MI & $42(27.8)$ & $148(15.2)$ & 1.97 & $(1.40-2.78)$ & & & $\longrightarrow$ & $<0.001$ \\
\hline Spontaneous MI & $39(26.3)$ & $108(11.4)$ & 2.62 & $(1.82-3.78)$ & & & $\longrightarrow$ & $<0.001$ \\
\hline Procedure-related MI & $6(3.6)$ & $47(4.6)$ & 0.80 & $(0.34-1.88)$ & & & & 0.610 \\
\hline \multirow[t]{3}{*}{ Revascularisation } & $119(76.2)$ & $734(71.8)$ & 1.02 & $(0.84-1.23)$ & & & & 0.871 \\
\hline & & & & $\Gamma$ & 1 & & 1 & \\
\hline & & & & 0.25 & 0.50 & $\begin{array}{l}1.0 \\
\mathrm{HR}\end{array}$ & 2.0 & \\
\hline
\end{tabular}

Ten-year clinical outcomes of non-ST-segment elevation acute coronary syndrome patients with or without DM. Cl, confidence interval; DM, diabetes mellitus; HR, hazard ratio; MI, myocardial infarction.

Table 1 Kaplan-Meier estimates of 10-year clinical outcomes in non-ST-segment elevation acute coronary syndrome patients with or without diabetes mellitus

\begin{tabular}{|c|c|c|c|c|c|c|c|c|c|}
\hline & \multicolumn{4}{|c|}{ Patients with diabetes mellitus $(n=166)$} & \multicolumn{4}{|c|}{ Patients without diabetes mellitus $(n=1034)$} & \multirow[b]{2}{*}{$\begin{array}{c}\text { Interaction } \\
P \text { value }\end{array}$} \\
\hline & EIS $(n=86)$ & SIS $(n=80)$ & $\begin{array}{c}\text { Hazard ratio } \\
(95 \% \mathrm{Cl})\end{array}$ & $P$ value & EIS $(n=518)$ & SIS $(n=516)$ & $\begin{array}{c}\text { Hazard ratio } \\
(95 \% \mathrm{Cl})\end{array}$ & $P$ value & \\
\hline Death or spontaneous MI & 49 (58.5) & $35(44.4)$ & $1.44(0.93-2.22)$ & 0.101 & $150(29.7)$ & $133(26.6)$ & $1.12(0.89-1.42)$ & 0.328 & 0.316 \\
\hline Death or MI & $50(59.6)$ & $36(45.7)$ & $1.46(0.95-2.25)$ & 0.081 & $172(34.0)$ & $141(28.1)$ & $1.26(1.01-1.58)$ & 0.041 & 0.561 \\
\hline All-cause death & $42(51.4)$ & $30(37.8)$ & $1.50(0.94-2.39)$ & 0.093 & $114(22.6)$ & $108(21.4)$ & $1.06(0.82-1.38)$ & 0.664 & 0.202 \\
\hline Cardiovascular death & 26 (36.9) & $22(28.8)$ & $1.26(0.71-2.22)$ & 0.428 & $71(14.8)$ & $63(13.0)$ & $1.13(0.81-1.59)$ & 0.475 & 0.741 \\
\hline Non-cardiovascular death & $16(23.1)$ & $8(12.7)$ & $2.15(0.92-5.02)$ & 0.078 & $43(9.2)$ & $45(9.7)$ & $0.96(0.63-1.46)$ & 0.849 & 0.093 \\
\hline MI & $21(27.0)$ & $21(28.1)$ & $1.01(0.55-1.84)$ & 0.982 & $85(17.6)$ & $63(12.9)$ & $1.39(1.00-1.93)$ & 0.047 & 0.362 \\
\hline Spontaneous MI & $19(25.7)$ & $20(26.8)$ & $0.94(0.50-1.76)$ & 0.839 & $56(11.9)$ & $52(10.8)$ & $1.07(0.74-1.56)$ & 0.716 & 0.747 \\
\hline Procedure-related MI & $5(5.8)$ & $1(1.3)$ & $4.79(0.56-41.03)$ & 0.153 & $34(6.6)$ & $13(2.5)$ & $2.67(1.41-5.07)$ & 0.003 & 0.610 \\
\hline Revascularisation & $68(83.8)$ & $51(68.2)$ & $2.12(1.47-3.07)$ & $<0.001$ & $428(83.2)$ & $306(60.4)$ & $2.62(2.26-3.04)$ & $<0.001$ & 0.261 \\
\hline
\end{tabular}

$\mathrm{Cl}$, confidence interval; EIS = early invasive strategy; MI, myocardial infarction; SIS, selective invasive strategy.

in other outcome measures in diabetic patients treated with an early or selective invasive strategy, except for a higher rate of revascularisation in the early invasive group [83.8\% vs. $68.2 \%$ (HR: 2.12; 95\% CI: $1.47-3.07$; $P<0.001)]$. There was no significant interaction between the presence of DM and treatment strategy for any of the study outcomes.

In a Cox proportional hazards model that adjusted for age, there was no significant difference in death or spontaneous MI at 10 years between diabetic patients treated with an early or selective invasive strategy (HR: 1.13; 95\% CI: $0.72-1.75 ; P=0.603)$. Adjustment for age $>65$ years (HR: 1.24 ; $95 \%$ CI: $0.80-1.92 ; P=0.346$ ) or age $>70$ year demonstrated similar results (HR: $1.18 ; 95 \%$ CI: $0.76-1.84 ; P=0.463$ ).

In this analysis of high-risk NSTE-ACS patients with elevated cardiac troponin, patients with DM had worse
10 -year outcomes compared to patients without DM. We could not demonstrate a statistically significant difference in 10-year death or spontaneous MI between the two strategies. This finding remained unchanged after adjustment for age. Our findings remain limited due to the small sample size, with only 166 diabetic patients. We did not record insulin-dependency of DM patients in our study and did not collect information on the development of DM during follow-up. Contemporary improvements such as drug-eluting stents, potent P2Y12 inhibitors, and radial access were not in use at the time of inclusion. Our finding illustrate the continuing longterm adverse effect of DM in NSTE-ACS. Although observed in other studies, non-cardiovascular death was also increased by two-fold in diabetics, suggesting unidentified confounding. Research to identify and improve treatment strategies for diabetic NSTE-ACS patients are warranted. 


\section{Acknowledgements}

This work was supported by the Interuniversity Cardiology Institute of the Netherlands (ICIN), the Working Group on Cardiovascular Research of the Netherlands (WCN), and educational grants from Eli Lilly, Sanofi/Synthelabo, Sanofi-Aventis, Pfizer, and Medtronic. Roche Diagnostics, the Netherlands, provided the reagents for Core Laboratory cardiac troponin T measurements.

\section{Conflicts of interest}

There are no conflicts of interest.

\section{References}

1 Damman P, Jernberg T, Lindahl B, de Winter RJ, Jeppsson A, Johanson $\mathrm{P}$, et al. Invasive strategies and outcomes for non-ST-segment elevation acute coronary syndromes: a twelve-year experience from SWEDEHEART. Eurointervention 2016; 12:1108-1116.
2 Angiolillo DJ. Antiplatelet therapy in diabetes: efficacy and limitations of current treatment strategies and future directions. Diabetes Care 2009; 32:531-540.

3 Amsterdam EA, Wenger NK, Brindis RG, Casey DE Jr, Ganiats TG, Holmes DR Jr, et al. 2014 AHA/ACC guideline for the management of patients with non-ST-elevation acute coronary syndromes: a report of the American College of Cardiology/American Heart Association Task Force on practice guidelines. J Am Coll Cardiol 2014; 64:e139-e228.

4 Roffi M, Patrono C, Collet JP, Mueller C, Valgimigli M, Andreotti F, et al.; ESC Scientific Document Group. 2015 ESC guidelines for the management of acute coronary syndromes in patients presenting without persistent ST-segment elevation: task force for the management of acute coronary syndromes in patients presenting without persistent ST-segment elevation of the European Society of Cardiology (ESC). Eur Heart J 2016; 37:267-315.

5 O'Donoghue ML, Vaidya A, Afsal R, Alfredsson J, Boden WE, Braunwald E, et al. An invasive or conservative strategy in patients with diabetes mellitus and non-ST-segment elevation acute coronary syndromes: a collaborative meta-analysis of randomized trials. J Am Coll Cardio/ 2012; 60:106-111.

6 de Winter RJ, Windhausen F, Cornel JH, Dunselman PH, Janus CL, Bendermacher PE, et al.; Invasive versus Conservative Treatment in Unstable Coronary Syndromes (ICTUS) Investigators. Early invasive versus selectively invasive management for acute coronary syndromes. $N$ Engl J Med 2005; 353:1095-1104. 